\title{
Seasonal Time Series Forecasting Models based on Artificial Neural Network
}

\author{
Benkachcha.S, \\ Laboratory LISER, ENSEM, \\ Km 7 BP 8118 Route El Jadida \\ Casablanca, Morocco
}

\author{
Benhra.j, \\ Laboratory LISER, ENSEM, \\ Km 7 BP 8118 Route El Jadida \\ Casablanca, Morocco
}

\author{
El Hassani.H, \\ École Hassania des Travaux \\ Publics Km 7 Route El Jadida \\ BP 8108 Casablanca, Maroc
}

\begin{abstract}
Forecasting is the starting point for drawing good strategies facing the demand variability in the increasingly complex and competitive today's markets. This article discusses two methods of dealing with demand variability in seasonal time series using artificial neural networks (ANN). First a multilayer perceptron model for time series forecasting is proposed. Several learning rules used to adjust the ANN weights have been evaluated. Secondly a causal method based on artificial neural networks, using the components of decomposed time series as input variables, has been used. The results show that ANNs yield almost the same accuracy with or without decomposition of the original time series.
\end{abstract}

\section{General Terms}

Feedforward Neural Networks; Multilayer Perceptron; Levenberg-Marquardt backpropagation algorithm. Seasonal time series forecasting model and causal method.

\section{Keywords}

Demand Forecasting, Supply Chain, Seasonal Time Series, Causal Method, Artificial Neural Networks (ANN).

\section{INTRODUCTION}

Market needs, demand variability and complexity of today's supply chains require an effective demand management as an important key to master the flow dynamics throughout the supply chain. Demand forecasting plays an important role for managing integrated logistics systems. It provides valuable information for several basic logistics activities including purchasing, inventory management, and transportation. The development of accurate and reliable forecast is the starting point for drawing good strategies facing the demand variability and uncertainty in the market. In practice most of the time series of sales have a seasonal component that must estimate and predict accurately [1].

Actually there are extensive forecasting techniques available for anticipating the future. This article discusses the use of two approaches based on neural networks to improve the quality of forecasts of time series with a seasonal component. The first approach considers implicitly the trend and seasonality, and is based only on the history of the variable to predict. The second takes into account explicitly the components of seasonality and trend as well as the irregularity. The three components are used as the inputs of the neural network and the output represents the demand.

The forecasts derived from the two approaches were compared and conclusions are drawn about the need to detrending and deseasonalizing the data before forecasting the time series using neural networks. The results show that the neural networks, with the right configurations, give almost the same accuracy with or without decomposition of the time series. Taking into account the cost factor, the method without decomposition is preferred due to its speed and lower cost.

The rest of the paper is organized as follows. Section 2 reviews the literature in forecasting and the use of Artificial Neural network in this area. Section 3 presents two prediction models based on neural networks. Section 4 discusses the results obtained using this methodology in a case study. Section 5 gives the conclusion of the paper.

\section{LITERATURE REVIEW}

Quantitative forecasting models can be grouped into two categories: the time series models and causal methods. Time series analysis tries to determine a model that explains the historical demand data and allows extrapolation into the future to provide a forecast in the belief that the demand data represent experience that is repeated over time.

This category includes naïve method, moving average, trend curve analysis, exponential smoothing, and the autoregressive integrated moving averages (ARIMA) models. These techniques are appropriate when we can describe the general patterns or tendencies, without regard to the factors affecting the variable to be forecast [2]. Easy to develop and implement, times series models are preferred for their having been used in many applications such as: Economic Forecasting, Sales Forecasting, Budgetary Analysis, Stock Market Analysis, Process and Quality Control and Inventory Studies [3].

Although in many forecasting applications the ARIMA model has been successfully used to predict seasonal time series, it suffers from limitation because of its linear form. However, due to its linearity, ARIMA is not always suitable for complex real-world problems [4]

To overcame this limitation many of artificial intelligence (AI) models such as nonlinear regression model, artificial neural networks (ANN), support vector machines (SVM) and genetic algorithm (GA) have been used to provide powerful nonlinear solutions to forecasting problems [5]. Coskun Hamzacebi proposed an artificial neural network (ANN) structure for seasonal time series forecasting. The comparison of results found by the proposed model and the traditional statistical models shows that ANN model comes with lower prediction error than other methods [6].

In pratice, many economic time series shows both seasonal nature and nonlinear characteristic of variations. As a decomposed method, the seasonal autoregressive integrated moving average (SARIMA) model can be formed to show the relationship between corresponding observation values of consecutive seasons [6]. F.-M. Tseng et al. used a hybrid 
model to forecast a seasonal time series. It was a combination of SARIMA and the neural network back propagation (BP) model. The results show that the combined model performs better forecasts than either the SARIMA model or the neural network by itself [7].

Mitrea, C. A., C. K. Lee, M. and Wu, Z. compared different forecasting methods like Moving Average (MA) and Autoregressive Integrated Moving Average (ARIMA) with Neural Networks (NN) models as Feed-forward NN and Nonlinear Autoregressive network with eXogenous inputs (NARX). The results have shown that forecasting with $\mathrm{NN}$ offers better predictive performances [8].

After being properly configured and trained by historical data, artificial neural networks (ANNs) can be used to approximate accurately any measurable function. Because they are fast and accurate, several researchers use ANNs to solve problems related to demand forecasting. The essence of the approach is to learn the patterns hidden in data and make generalizations of these patterns. ANN provides outstanding results even in the presence of noise or missing information [9].

\section{METHODOLOGIE}

\subsection{Decomposition model with seasonal adjustment}

Most time series consist of combination of three components: trend cycle behavior (TS), seasonal effects (S) and irregular fluctuations (IR). Usually we choose one of the two following models to describe how these components could fit together in a time series:

$$
\text { Additive model: } \quad Y=T C+S+I R
$$

Multiplicative model: $Y=T C \times S \times I R$

Where $Y$ is the original series, $T C$ is the trend-cycle, $S$ is the seasonal component, and $I R$ is the irregular component.

Seasonal decomposition is a process of estimating the seasonal component called seasonal factors using generally a series of moving averages and smoothing calculations to decompose the original series into trend, seasonal, and irregular components [10].

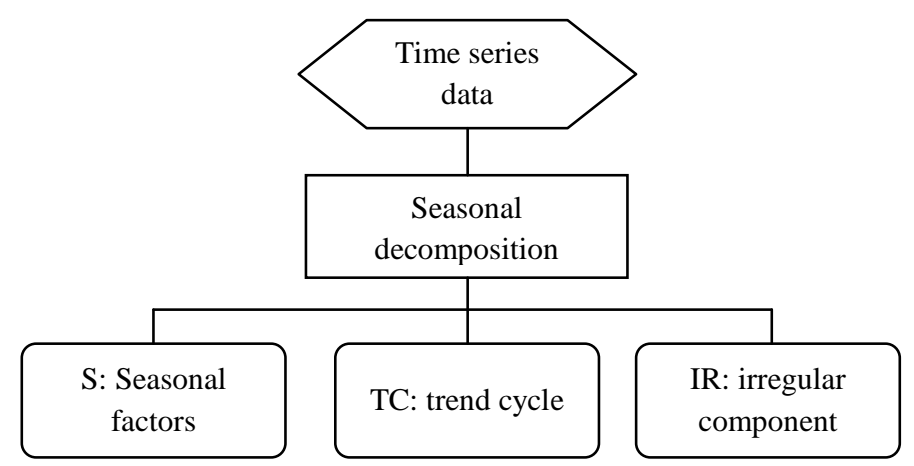

Fig.1 Decomposition of the original series into trend, seasonal, and irregular components

Once the seasonal factors are defined, the original series is divided (for multiplicative models) or subtracted (for additive models) by seasonal factors to find the deseasonalized series which can be extrapolated to determine the forecasted demand.

\subsection{Multilayer perceptrons (MLP)}

The MLP (multilayer perceptrons) are the most popular type of Feedforward neural network used in approximation and optimization problems. Organized in layers, an MLP allow unidirectional signal flow from inputs to the outputs [11]. An example of multilayer perceptrons is shown in figure 1. It consists of an input layer, a hidden layer, and an output layer.

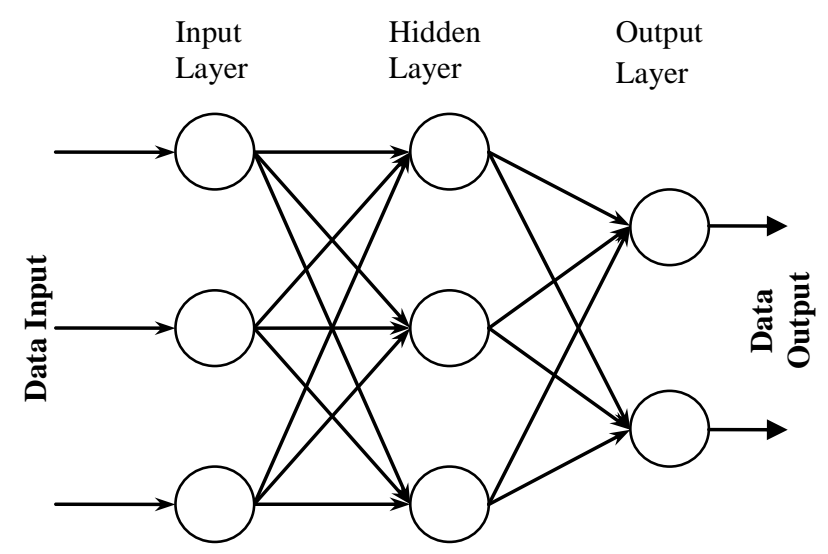

Fig.2 Basic structure of Multilayer Perceptrons

A single-input neuron is shown in Figure 2. It consists of a processing unit which contains weights $\left(w_{i j}\right)$ and summing function followed by a transfert function $(f)$.

The summer output, referred to as the total synaptic input of the neuron, is given by the inner product of the input and weight vectors:

$$
n_{i}=\sum w_{i j} x_{j}
$$

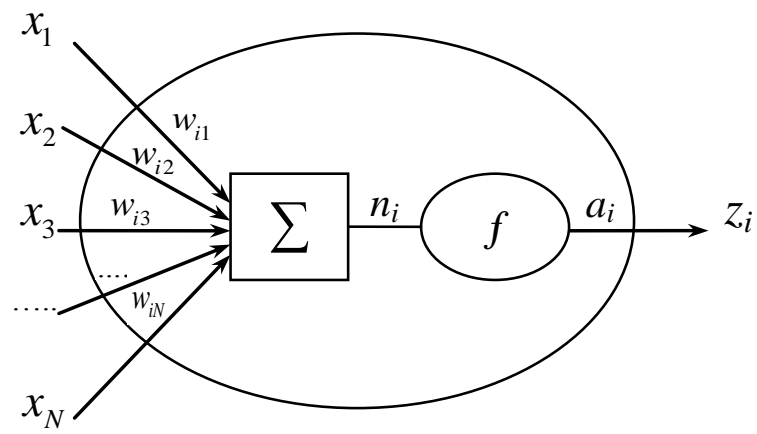

Fig.3 Single-input neuron : $w_{i j}, n_{i}, f$ and $a_{i}$ are a neural network elements 
The transfer function is chosen related to the problem that the neuron is attempting to solve :

- For a non-linear hypothesis a sigmoid function :

$f(k)=1 / 1+e^{-k}$,

having a range of $[0,1]$, can be used.

- The hyperbolic-tangent function :

$f(k)=e+{ }^{k}+e^{-k} / e+{ }^{k}+e^{-k}$

is suitable for non-linear problems with positive and negative outputs.

The output value (or activation) of the neuron is given by:

$$
a_{i}=f\left(n_{i}\right)=f\left(\sum w_{i j} x_{j}\right)
$$

Where:

- $f\left(n_{i}\right)$ is the transfer function,

- $\quad w_{i j}$ is the connection weight between node $j$ and node $i$,

- $\quad x_{j}$ the input signal from the node $j$.

- $\quad a_{i}$ is the output of the neuron $i$,

The general process responsible for training the network is mainly composed of three steps: feed forward the input signal, back propagate the error and adjust the weights.

The back-propagation algorithm try to improve the performance of the neural network by reducing the total error which can be calculated by:

$$
E=\frac{1}{2} \sum_{p} \sum_{j}\left[o_{j p}-d_{j p}\right]^{2}
$$

Where $E$ is the square error, $p$ the number of applied patterns, $d_{j p}$ is the desired output for $j$ th neuron when $p$ th pattern is applied and $\boldsymbol{O}_{j p}$ is the $j$ th neuron output.

\subsection{Time series and multilayer perceptron- based model}

The extrapolative or time series forecasting model is based only on values of the variable being forecast. Thus for the multilayer perceptron used to forecast the time series the inputs are the past observations without removing the seasonal effect from the data series and the output is the future value. The MLP performs the following function mapping:

$\hat{y}_{t}=f\left(y_{t-1}, y_{t-2}, \cdots, y_{t-n}\right)$

Where $\hat{y}_{t}$ is the estimated output, $\left(y_{t-1}, y_{t-2}, \cdots, y_{t-n}\right)$ is the training pattern which consist of a fixed number (n) of lagged observations of the series. With $\mathrm{P}$ observations for the series, we have P-n training patterns.

There is no suggested systematic way to determine the number of the input nodes (n). After several essays this number has been fixed successfully at 24 nodes for the case studied next for the airline passenger.

The neural network architecture can be shown in figure 4 . Other design parameters as training algorithm will be discussed and selected in the next paragraph.

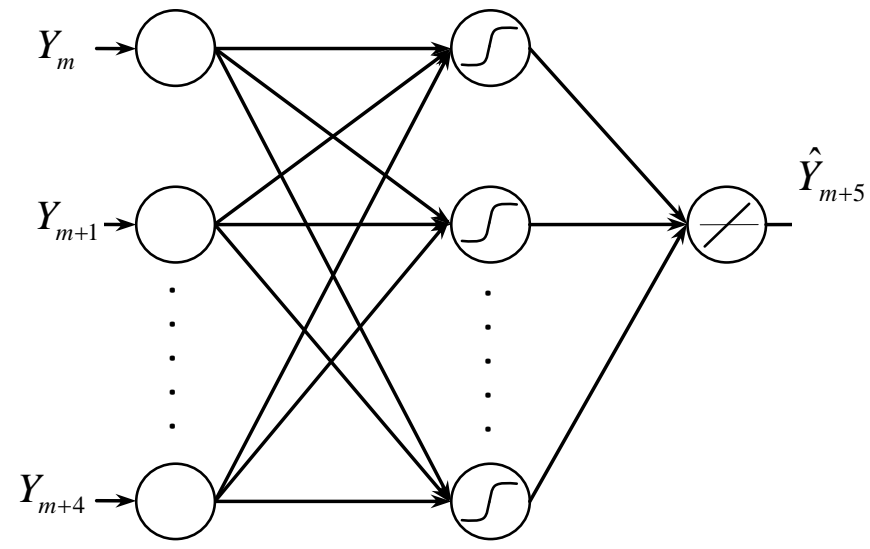

Fig.4 Multilayer perceptron for time series forecasting

\subsection{Causal method based on artificial neural networks}

\subsubsection{The network architecture}

The neural network architecture is composed of three input nodes (corresponding to the three components of seasonal time series: TC, $S$ and $I R$ ), one output node, and an appropriate number of hidden nodes. The most common approach to select the optimal number of hidden nodes is via experiment or by trial-and-error [12]. We use the same approach to define the number of neurons in the hidden layer.

One other network design decisions include the selection of activation functions, the training algorithm, learning rate and performance measures. For non-linear prediction problem, a sigmoid function at the hidden layer, and the linear function in the output layer are generally used. The best training algorithm is the levenberg-Marquardt back-propagation [13]

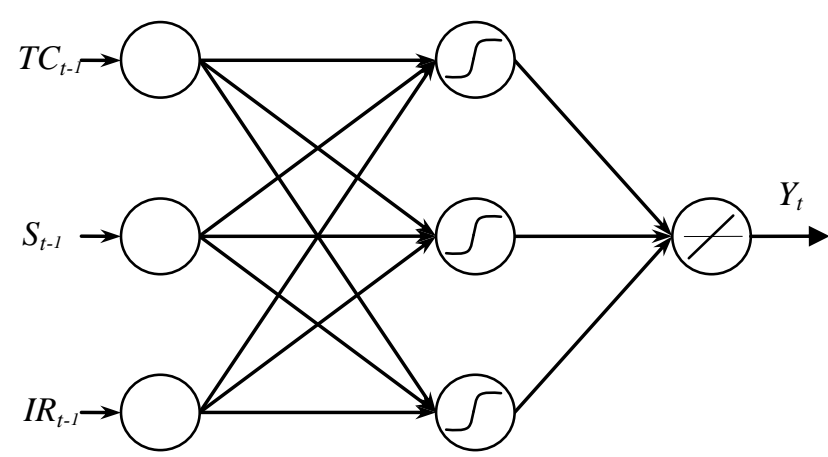

Fig.5 Causal method and multi-layer perceptron

\section{RESULTS AND DISCUSSIONS}

\subsection{The airline passenger data set}

The airline passenger (AP) data set was used by Faraway and Chatfield to obtain the best ANN estimator [14]. The airline passenger data set shows the airline passenger numbers between January 1949 and December 1960 on a monthly basis. 


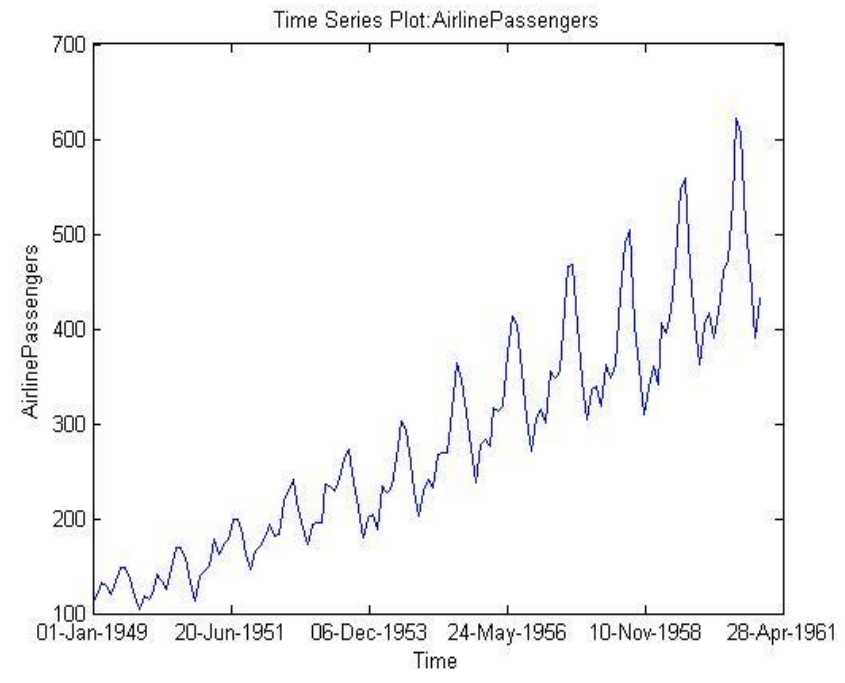

Fig. 6 Airline passenger data from 1949 to 1960

As shown in Fig. 7, the original series is decomposed into trend, seasonal, and irregular components.

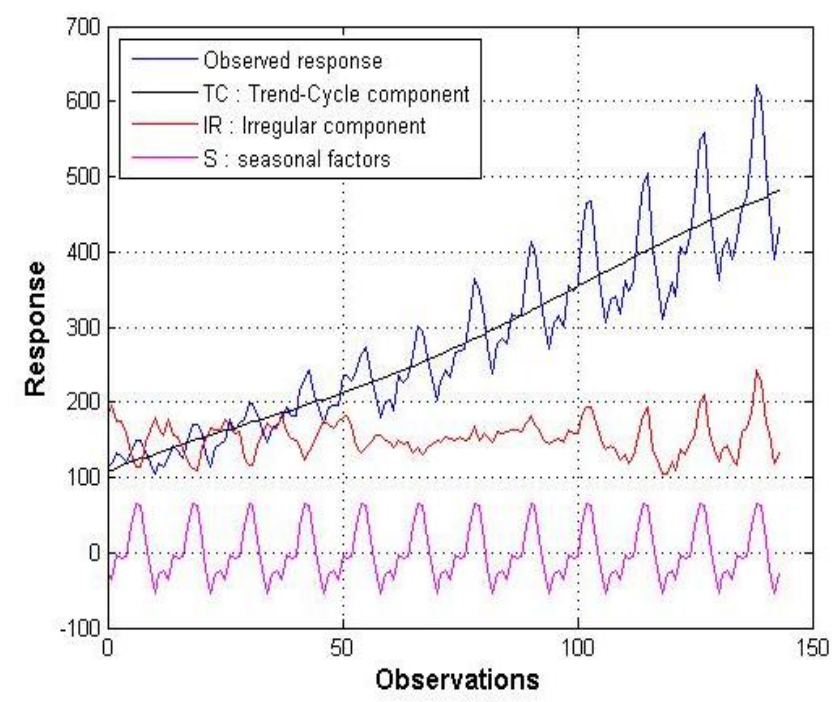

Fig.7 Seasonal decomposition of AP data set

\subsection{Time series forecasting model}

Neural Network Toolbox provides a complete environment to design, train, visualize, and simulate neural networks.

For the neural network training method, we try three algorithms to improve network performance:

- The backpropagation (BP) algorithm: It is a gradient steepest descent method, implemented with traingd function in toolbox of MATLAB.

- Gradient descent with adaptive learning rate backpropagation (BPA): traingda function in MATLAB toolbox.

- Levenberg-Marquardt backpropagation (LM) : trainlm function in MATLAB toolbox.

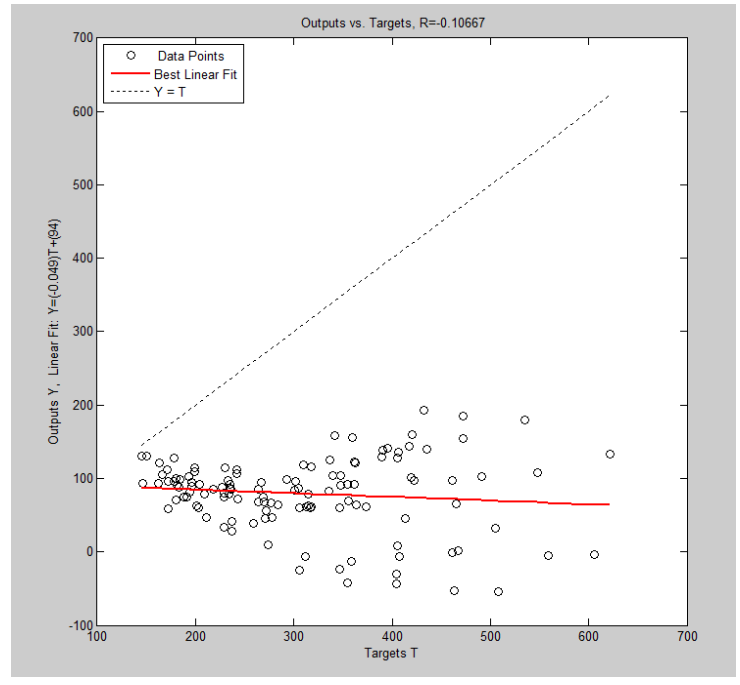

Fig.8 Plot Regression for AP time series forecast model with $B P$ training algorithm

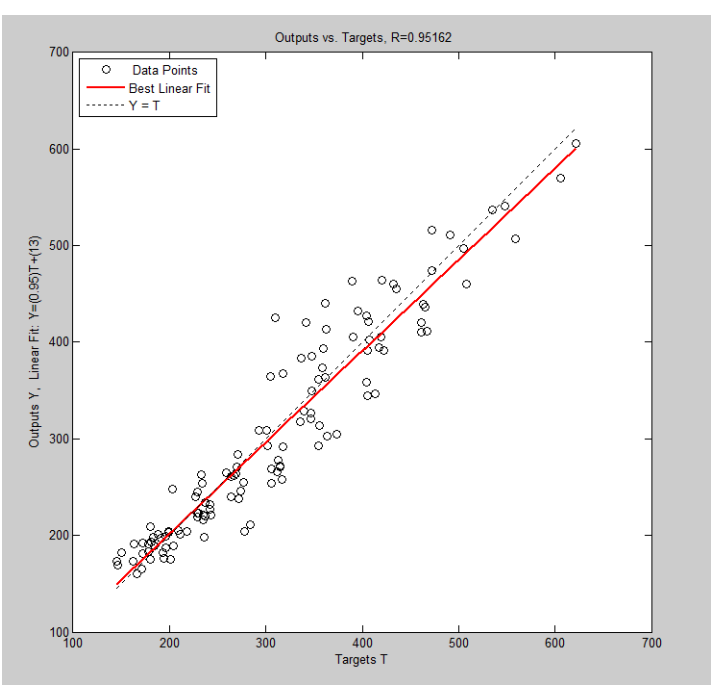

Fig.9 Plot Regression for AP time series forecast model with BPA training algorithm

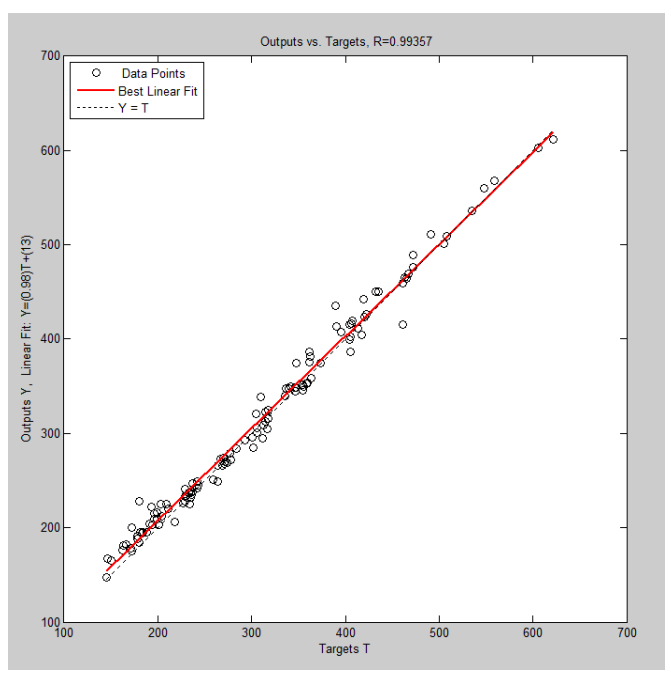

Fig.10 Plot Regression for AP time series forecast model with $\mathrm{LM}$ training algorithm 
The plot regression for the three training algorithms in last figures has shown that with the same topology, LM algorithm gives better solution than those found using BP or BPA algorithms.

BP algorithm is the most popular method in training neural networks. However it has a low training efficiency because of its slow convergence. The BPA algorithm uses a dynamic learning rate to increase the training speed of BP.

Even if the BPA algorithm uses a dynamic learning coefficient, its performance remains lower than LM, which takes a second order function as propagation algorithm.

The LM algorithm can estimate the learning rate in each direction of gradient using Hussian matrix [15].

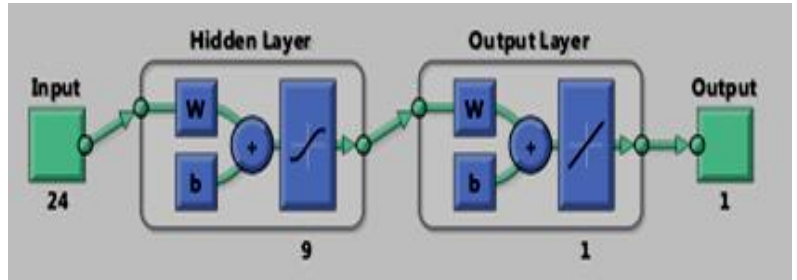

Fig.11 Neural network training for APTS forecast model (LM algorithm)

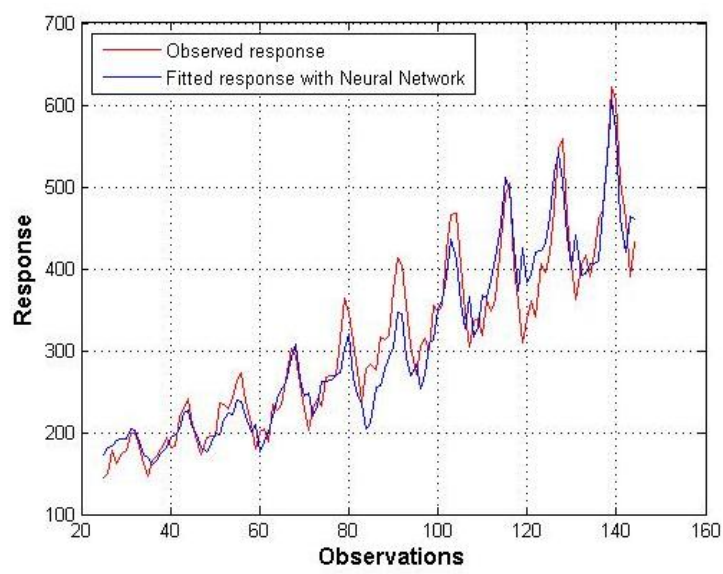

Fig.12 AP Demand forecasting with time series model based neural network (BPA training algorithm)

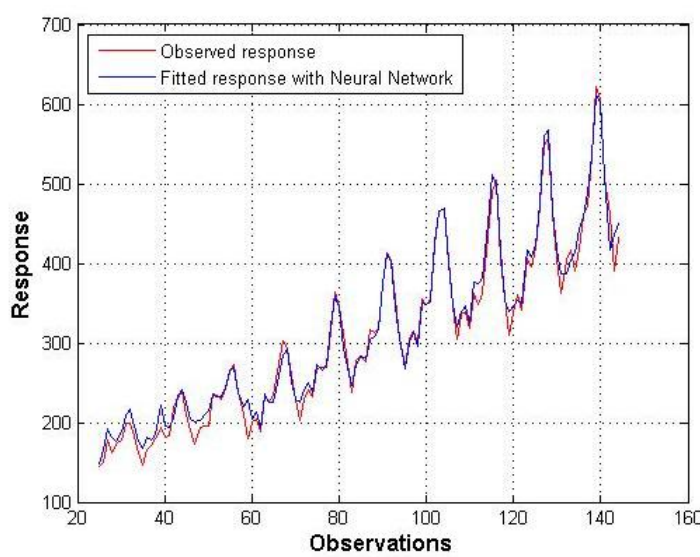

Fig.13 AP Demand forecasting with time series model based neural network (LM training algorithm)

The figures 11 and 12 illustrate that a better accuracy is given by using LM as the training algorithm.

\subsection{Causal model}

For the causal model we use the function newff to create a feed forward neural network. The Levenberg-Marquardt back propagation algorithm is implemented. "trainlm" is a network training function that updates weights according to Levenberg-Marquardt optimization [16]. The obtained response with this architecture is given in the figure below:

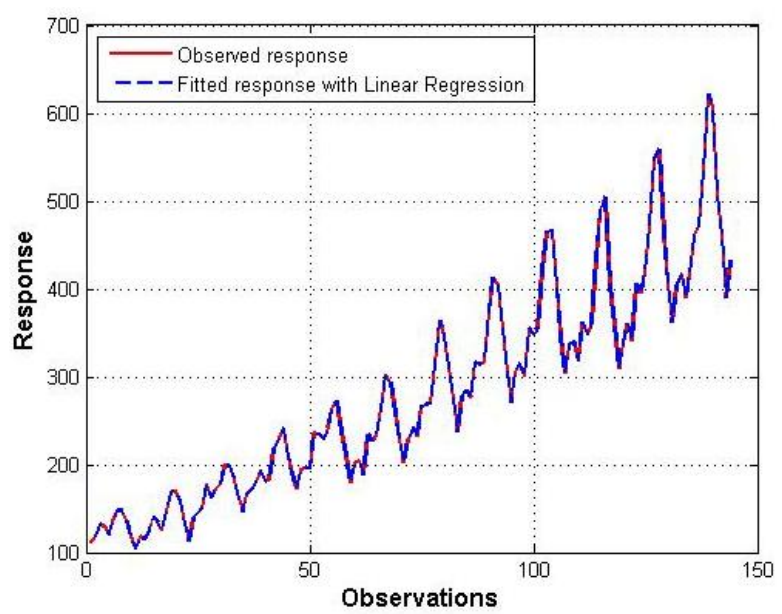

Fig.14 AP time series trained with causal forecast model based neural network (LM training algorithm)

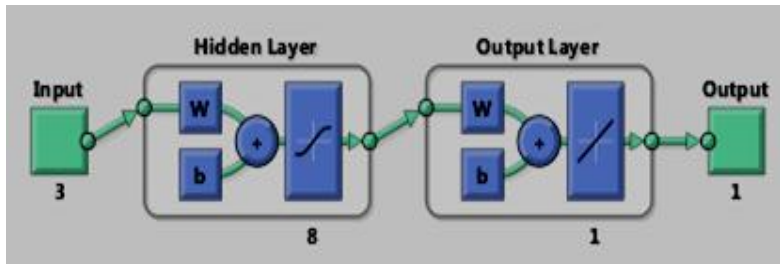

Fig.15 Neural network training for causal forecast model (for AP time series)

\subsection{Discussion of results}

With the selection of appropriate parameters such as the number of neurons in each layer and selection of the best back-propagation algorithm, the two neural models: time series and causal method, gives satisfactory results.

However, if we take into consideration the cost of the prediction method, the time series model will be chosen since it takes into consideration only the history of the variable to predict.

\section{CONCLUSION}

Demand forecasting plays a crucial role in the supply chain of today's company. Among all forecasting methods, neural networks models are capable of delivering the best results if they are properly configured. Two approaches based on multilayer perceptron have been developed to predict demand: time series model and causal methods. The best training algorithm is the Levenberg-Marquardt backpropagation algorithm. The number of hidden layers and the number of neurons in each layer depends on the chosen method and case study. With a judicious choice of the architecture and parameters of the neural network, both approaches have yielded good results. However, the cost of the prediction method parameter allows us to prefer the time series model since it takes into consideration only the history of the variable to predict. 


\section{REFERENCES}

[1] Benkachcha. S, Benhra. J, El Hassani. H, 2013. Causal Method and Time Series Forecasting model based on Artificial Neural Network. International Journal of Computer Applications. Vol. 75, No 7, p.0975 - 8887.

[2] Kesten C. Green, J. Scott Armstrong 2012. Demand Forecasting: $\quad$ Evidence-based Methods. https://marketing.wharton.upenn.edu/profile/226/printFri endly.

[3] Gosasang, V., Chan., W. and KIATTISIN, S. 2011. A Comparison of Traditional and Neural Networks Forecasting Techniques for Container Throughput at Bangkok Port. The Asian Journal of Shipping and Logistics, Vol. 27, $\mathrm{N}^{\circ} 3$, pp. 463-482.

[4] Zhang G.P. 2003. Time series forecasting using a hybrid ARIMA and neural network model. Neurocomputing Vol. 50, p 159-175.

[5] Shuai Wang, Lean Yu, Ling Tang, Shouyang Wang, 2011. A novel seasonal decomposition based least squares support vector regression ensemble learning approach for hydropower consumption forecasting in China. Energy Vol.36, p. 6542-6554.

[6] Coskun Hamzaçebi 2008. Improving artificial neural networks' performance in seasonal time series forecasting. Vol.178, p 4550-4559.

[7] Tseng F.-M. , Yu H.-C., Tzeng G.-H. 2002. Combining neural network model with seasonal time series ARIMA model. Technological Forecasting \& Social Change Vol. 69 , p. $71-87$.

[8] Mitrea, C. A., Lee, C. K. M., WuZ. 2009. A Comparison between Neural Networks and Traditional Forecasting Methods: A Case Study". International Journal of Engineering Business Management, Vol. 1, No. 2, p 1924.
[9] Daniel Ortiz-Arroyo, Morten K. Skov and Quang Huynh, 2005 . Accurate Electricity Load Forecasting With Artificial Neural Networks. Proceedings of the 2005 International Conference on Computational Intelligence for Modelling, Control and Automation, and International Conference on Intelligent Agents, Web Technologies and Internet Commerce (CIMCAIAWTIC'05),

[10] Shuai Wang, Lean Yu, Ling Tang, Shouyang Wang. 2011. A novel seasonal decomposition based least squares support vector regression ensemble learning approach for hydropower consumption forecasting in China. Energy Vol. 36, p. 6542-6554.

[11] Wilamowski B. M. 2011. Neural Network Architectures. Industrial Electronics Handbook, vol. 5 - Intelligent Systems, 2nd Edition, chapter 6, pp. 6-1 to 6-17, CRC Press.

[12] Zhang G., Patuwo, B. E., Hu, M.Y. 1998. Forecasting with artificial neural networks : The state of the art. International Journal of Forecasting.Vol.14, p 35-62.

[13] Norizan M., Maizah H. A., Suhartono, Wan M. A. 2012. Forecasting Short Term Load Demand Using Multilayer Feed-forward (MLFF) Neural Network Model. Applied Mathematical Sciences, Vol. 6, no. 108, p. 5359 - 5368

[14] Faraway J., Chatfield C. 1998. Time series forecasting with neural networks: a comparative study using the airline data. Applied Statistics. Vol. 47 p. 231-250.

[15] Wilamowski B. M., Yu H. 2010. Improved Computation for Levenberg Marquardt Training. IEEE Trans. on Neural Networks, vol. 21, no. 6, pp. 930-937.

[16] Anandhi V., ManickaChezian R., ParthibanK.T. 2012 Forecast of Demand and Supply of Pulpwood using Artificial Neural Network. International Journal of Computer Science and Telecommunications, Vol.3, Issue 6, June, pp. 35-38. 